\title{
Implantação de Melhoria de Processo de Software no Tribunal Superior Eleitoral
}

\author{
Cybele Caldeira Macedo ${ }^{1}$, Sandra Helena Camargo de Lima ${ }^{1}$, Ana Regina Rocha ${ }^{2}$, \\ Ana Candida Natali ${ }^{2}$, Káthia Marçal de Oliveira ${ }^{3}$, Paula Mian ${ }^{2}$,Andrea Barreto ${ }^{2}$, \\ Ahilton Barreto ${ }^{2}$, Gleison Santos ${ }^{2}$, Tayana Conte ${ }^{2}$ \\ ${ }^{1}$ Tribunal Superior Eleitoral - Coordenação de Sistemas Eleitorais - Brasília, DF \\ ${ }^{2}$ COPPE/UFRJ - Rio de Janeiro, RJ \\ ${ }^{3}$ Universidade Católica de Brasília - Brasília, DF \\ \{cmacedo, sandrah \}etse.gov.br; \{darocha, anatali, pgmian, ansoares, \\ ahilton, gleison, tayana\}dcos.ufrj.br; kathiaducb.br
}

\begin{abstract}
Software process improvement is the goal of many organizations that aim at achieving better product quality and market recognition through international organizations official appreciation. Being aware of this fact, Electoral System Department of the Brazilian Superior Electoral Court realized importance of implementing software process improvement procedures in order to standardize software development in all its electoral systems development units. This article presents an experience report on this process improvement program implementation that culminated in the maturity and capability model CMMI level 2 evaluation after a six months work period.
\end{abstract}

Resumo. Melhoria de processo de software tem sido uma meta constante das instituições em busca da melhor qualidade dos produtos gerados $e$ do reconhecimento do mercado vindo de avaliações oficiais de órgãos internacionais. Ciente dessa realidade, a Coordenadoria de Sistemas Eleitorais do Tribunal Superior Eleitoral percebeu a necessidade de implantar procedimentos para melhoria dos seus processos de desenvolvimento em busca de uma padronização nas diferentes unidades de desenvolvimento de sistemas eleitorais. Este artigo apresenta um relato de experiência sobre implantação desse programa de melhoria de processo que culminou na avaliação nível 2 do modelo de maturidade e capacidade CMMI após pouco mais de seis meses de trabalho.

\section{Introdução}

Ter processos definidos e institucionalizados tem se tornado um desafio comum nas organizações que desenvolvem software em busca dos benefícios defendidos por modelos como o CMMI (Capability Maturity Model Integration) [Chrissis et al., 2003], tais como: prover uma linguagem comum para todos os participantes dos projetos, permitir a comparação entre projetos e estabelecer um caminho de melhoria contínua.

Objetivando alcançar tais benefícios a Coordenadoria de Sistemas Eleitorais do Tribunal Superior Eleitoral (CSE/TSE) decidiu pela implantação de um programa de melhoria de processos nas suas três unidades de desenvolvimento de sistemas eleitorais: Seção de Alistamento Eleitoral, Seção de Processamento de Eleições e Seção de Voto Informatizado. 
Em um primeiro momento, esse desafio poderia parecer desnecessário visto que a CSE/TSE já desenvolve sistemas capazes de processar com rapidez e segurança as eleições de um país de dimensões continentais, como o Brasil. Além disso, o trabalho desenvolvido pela CSE/TSE já adquiriu reconhecimento internacional, visto que o sistema eleitoral brasileiro é utilizado em diversos países, como Argentina, Republica Dominicana, Guatemala, entre outros. No entanto, a CSE/TSE desejava a padronização do desenvolvimento de software nas suas três unidades e ser reconhecido internacionalmente pela comunidade de software no que se refere ao desenvolvimento com qualidade. Para alcançar esse objetivo, a COPPE/UFRJ definiu uma estratégia de implantação e melhoria de processo de software, com apoio ferramental, que envolveu as três unidades de forma padronizada e customizada para a CSE/TSE. Essa estratégia utilizou como base os processos da ISO/IEC 12207 [ISO/IEC 12207, 1998] e do modelo CMMI [Chrissis et al., 2003].

$\mathrm{Na}$ próxima seção será apresentada brevemente a estrutura da CSE/TSE mostrando os tipos de sistemas desenvolvidos. Na seção 3, é apresentada a estratégia de implantação de melhoria de processo utilizada, descrevendo o processo definido e sua melhoria. Na seção 4, são descritas a definição e a melhoria do processo de software na CSE/TSE. Na seção 5 é apresentada a preparação para a avaliação oficial, e na seção 6, as conclusões e trabalhos futuros.

\section{A Coordenadoria de Sistemas Eleitorais do TSE}

O TSE é o órgão responsável pela organização e execução dos serviços eleitorais do governo federal que envolve todas as atividades do processo eleitoral: do cadastramento de eleitor e de partidos ao apoio logístico e informatizado para as eleições e processamento de votos, além da conservação das urnas eletrônicas. A maioria dessas atividades é apoiada por sistemas informatizados (atividades em cinza).

$\mathrm{Na}$ estrutura organizacional do TSE, a CSE está subordinada a Secretaria de Informática sendo apoiada pela Assessoria de Planejamento. A CSE é dividida em três unidades de desenvolvimento de sistemas eleitorais: Seção de Voto Informatizado (SVI), na qual os sistemas de voto são especificados e desenvolvidos, além de especificar o ambiente para a cabine de votação informatizada (sistema operacional, drivers, APIs); Seção de Alistamento Eleitoral (SAE), onde são desenvolvidos os sistemas de cadastramento de eleitores, partidos, afiliações partidárias, entre outros; e, Seção de Processamento de Eleições (SPE); onde são especificados e desenvolvidos sistemas específicos para eleição como propaganda eleitoral oficial, preparo da eleição e sumarização dos resultados.

Os sistemas desenvolvidos por essas unidades são definidos por grupos de trabalho constituídos no âmbito da justiça eleitoral e formado por representantes do TSE e dos Tribunais Regionais Eleitorais. Esses grupos de trabalho estabelecem critérios e normas para o desenvolvimento, testes e implantação de procedimentos para verificação do funcionamento dos sistemas eleitorais.

\section{Estratégia de Implantação do Programa de Melhoria de Processos de Software}

Apesar das diferentes unidades da CSE já desenvolverem software reconhecido internacionalmente, essas unidades trabalhavam de forma independente, sem 
padronização e sem o uso de um processo formal de software. Nesse contexto, percebeu-se a dificuldade de definir um processo realmente adequado a todas as unidades em um primeiro momento. Dessa forma, ao se definir a estratégia de implantação para a CSE/TSE, decidiu-se por:

- Definir e implantar rapidamente uma primeira versão do processo de desenvolvimento padrão baseado na norma ISO/IEC 12207 [ISO/IEC 12207, 1998] e nas áreas de processo do CMMI [Chrissis et al., 2003] nível 2.

- Usar apoio ferramental para implantação do processo de desenvolvimento e de processos de apoio de forma a estabelecer um ambiente comum de trabalho com o uso da Estação TABA [Rocha et al., 2005a] configurado para o TSE. Optou-se por adotar a Estação Taba, pois já havia sido constatado que este apoio ferramental pode ser adequado e bastante útil para disseminar as práticas introduzidas pelos processos de software e para agilizar os treinamentos em engenharia de software necessários para a execução dos processos de forma direcionada às necessidades da organização [Rocha et al., 2005b].

- Realizar um treinamento inicial em todas as áreas de processo do CMMI nível 2, no processo definido para a CSE/TSE, e o uso de mentoring para apoio na realização das atividades do processo e no uso das ferramentas;

- Estabelecer uma auditoria externa mensal que garantisse a conformidade na realização do processo e produtos de software; e,

- Melhorar continuamente o processo a partir de solicitações de mudanças no processo, resultados de avaliações, evolução para níveis mais altos de maturidade.

Para iniciar os trabalhos foram criados três grupos de apoio às unidades:

- Grupo de Gerência de Configuração - responsável por realizar auditoria de gerência de configuração dos projetos, acompanhar as seções para auditoria de gerência de configuração de código fonte e gerar mensalmente de relatórios de auditoria;

- Grupo de Medição e Análise - responsável por analisar as métricas organizacionais mensalmente, comunicar mensalmente aos gerentes e líderes o resultados das medições organizacionais, e comunicar trimestralmente à gerência de alto nível o resultados das medições organizacionais, e;

- Grupo de Garantia da Qualidade de Processo e de Produto (GQPP) responsável por avaliar os produtos e processos das três seções, avaliar a aderência dos processos de medição e análise e de gerência de configuração, comunicar a gerência de alto nível o status dos processos e receber as solicitações de mudanças nos processos e procedimentos. definiu-se:

Para garantir a padronização da realização das atividades nas três unidades,

- Políticas organizacionais referentes a todas as áreas de processo do nível 2 CMMI, para definir o objetivo de cada processo de apoio e de suas atividades, além de estabelecer quais produtos deveriam ser obrigatoriamente desenvolvidos, as interações com os grupos de apoio e as datas para execução de atividades e elaboração de relatórios mensais. 
- Diretrizes de projeto com o passo a passo de como realizar as diferentes atividades do processo de desenvolvimento.

\section{Definição e Melhoria do Processo de Software}

A definição e implantação de processos em uma organização devem ser feitas em etapas bem definidas e controladas, como um projeto: com recursos alocados, datas de início e fim definidas e responsabilidades atribuídas. Na CSE/TSE, para se obter a avaliação CMMI nível 2, o Projeto Rumo ao CMMI (como foi denominado pela organização) foi constituído de várias fases, conforme descrito na figura 1 .

\begin{tabular}{|c|c|c|c|c|c|c|c|c|c|}
\hline Nome da tarefa & Abr.05 & Maion:05 & Junn:05 & Jullo5 & Ago:05 & Set.05 & Outos & Novios & Dez/05 \\
\hline Contratação da Consultoria Externa & & & & & & & & & \\
\hline $\begin{array}{l}\text { Treinamentos para os Funcionários em Engenharia de } \\
\text { Software e áreas de Processo CMMM Nivel } 2\end{array}$ & & & & & & & & & \\
\hline Definiçăo da $1^{2}$ versã̃o do Processo (CMMl) & & & & & & & & & \\
\hline Treinamento no Processo & & & & & & & & & \\
\hline Formaçắo do Grupo de Processos & & & & & & & & & \\
\hline $\begin{array}{l}\text { Configuraçắo do Ambiente TABA para a } 1^{2} \text { Versăo do } \\
\text { Processo }\end{array}$ & & & & & & & & & \\
\hline Acompanhamento dos Projetos (Mentoring) & & & & & & & & & \\
\hline $\begin{array}{l}\text { Definição da } 2^{2} \text { versão do Processo após solicitações } \\
\text { melhoria (CMMI) }\end{array}$ & & & & & -9 & & & & \\
\hline $\begin{array}{l}\text { Configuraçäo do Ambiente TABA para a } 2^{2} \text { Versăo do } \\
\text { Processo }\end{array}$ & & & & & & & & & \\
\hline Avaliaçăo Prévia CMMl (Readiness Assesment) & & & & & & & & 回 & \\
\hline $\begin{array}{l}\text { Ajustes em Relaçăo ao Resultado da Avaliaçăo Prévia } \\
\text { (Readiness Assesment) }\end{array}$ & & & & & & & & & \\
\hline Avaliação Formal do SEl no CMMI Nivel 2 (SCAMPI) & & & & & & & & & 문 \\
\hline
\end{tabular}

Figura 1. Cronograma das atividades do Projeto Rumo ao CMMI

Para a definição do processo, optou-se por definir um processo padrão de desenvolvimento único que integrasse as diferentes áreas de processo do CMMI nível 2 conforme esquematizado na figura 2. Com o uso da primeira versão em alguns projetos, seria possível a identificação de oportunidades de melhoria no processo de forma a torná-lo mais adequado à organização e às necessidades específicas de seus projetos.

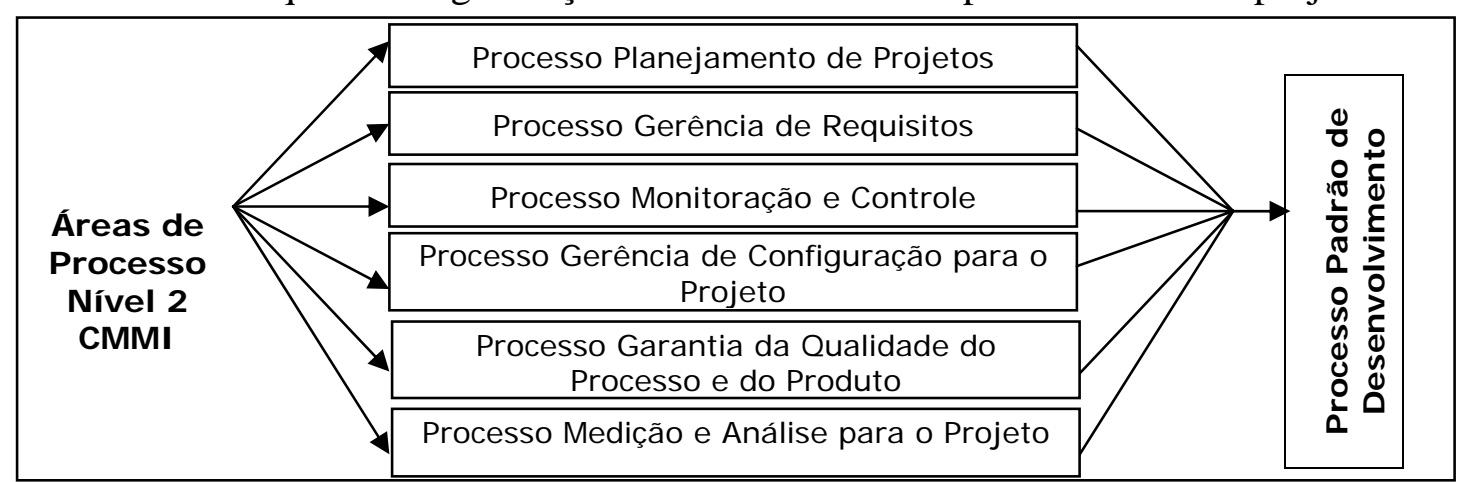

Figura 2. Processo Padrão de Desenvolvimento aderente ao CMMI nível 2

A primeira versão do processo era composta de sete grandes fases, conforme mostra a figura 3, e foi utilizada por projetos nas três unidades de desenvolvimento de sistemas eleitorais. Desta forma, o processo foi utilizado em projetos com as mais variadas características, e que representavam uma boa amostra dos projetos 
desenvolvidos pela CSE/TSE. Isso permitiu que fossem detectadas diversas oportunidades de melhoria no processo padrão segundo o ponto de vista das três unidades envolvidas, contribuindo na evolução para um processo que fosse mais adequado à organização como um todo. Foram ao todo oito projetos que utilizaram a primeira versão do processo.

Para solicitar mudanças no processo padrão, foi disponibilizado para todos os envolvidos (líderes de projeto, grupos de processos, equipe consultora, etc.) um formulário de solicitação de alteração no processo padrão, conforme ilustrado na figura 4. Esse formulário deveria ser preenchido e enviado ao GQPP. Periodicamente, as solicitações de mudança eram analisadas e decidia-se sobre quais mudanças deveriam ser efetivamente realizadas.

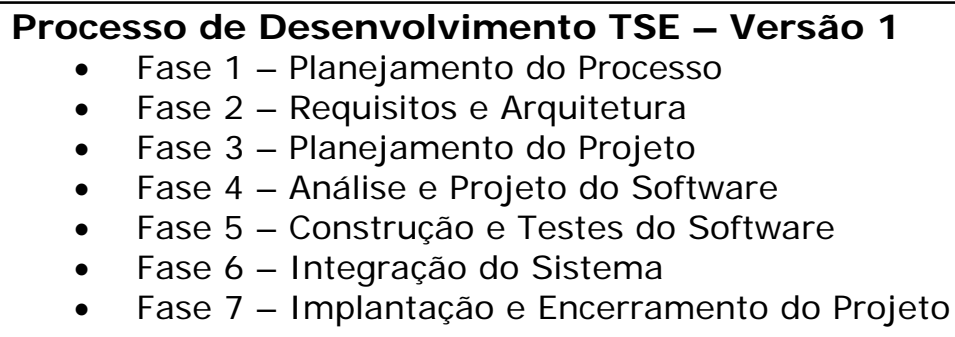

Figura 3. Processo Padrão de Desenvolvimento TSE - Versão 1

\begin{tabular}{|c|c|}
\hline Registrc & $\begin{array}{c}\text { Tribunal Superior Eleitoral - TSE } \\
\text { Secretaria de Informática - SI } \\
\text { Coordenadoria de Sistemas Eleitorais - CSE } \\
\text { de Solicitações de Alteração no Processo Padrão - TSE }\end{array}$ \\
\hline \multicolumn{2}{|r|}{ Solicitação } \\
\hline Solicitante: & Marta J uvina de Medeiros \\
\hline Data: & 17 de junho 2005 \\
\hline $\begin{array}{l}\text { Descrição da } \\
\text { Solicitação: }\end{array}$ & $\begin{array}{l}\text { Incluir na fase } 1 \text { do Processo Padrão atividades de Planejamento do Projeto } \\
\text { necessárias para executar a fase } 2 \text { e } 3 \text {. }\end{array}$ \\
\hline J ustificativa: & $\begin{array}{l}\text { Necessidade de realizar Planejamento da Organização e da Gerência de } \\
\text { Configuração e ter um cronograma inicial para a fase. }\end{array}$ \\
\hline \multicolumn{2}{|r|}{ Apreciação da Solicitação } \\
\hline Responsável: & Consultora Ana Regina Rocha e representante do GQPP Sandra Helena. \\
\hline Data: & 20 de junho 2005 \\
\hline Decisão: & Aceita \\
\hline $\begin{array}{l}\text { Modificação a ser } \\
\text { Realizada: }\end{array}$ & $\begin{array}{l}\text { Evoluir o processo padrão, para que na sua segunda versão seja incluída a } \\
\text { atividade de Planejamento Inicial do Projeto, contemplando as atividades } \\
\text { requeridas. }\end{array}$ \\
\hline
\end{tabular}

Figura 4. Formulário de Solicitação de Alteração no Processo Padrão

Cerca de dois meses depois do uso da primeira versão do processo nos projetos, já era possível identificar sugestões de modificação no processo de desenvolvimento padrão. Assim, foi definida a segunda versão do processo com base nessas sugestões. 
Foi também realizada uma nova configuração da Estação TABA para contemplar o novo processo, além da revisão das diretrizes existentes.

A segunda versão do processo também era composta por sete fases. As principais mudanças em relação à primeira versão do processo padrão foram:

- A primeira fase passou a considerar não apenas o planejamento do processo, mas o planejamento inicial do projeto, como definição da equipe, alocação de recursos (ferramentas etc), plano de gerência de configuração, e um cronograma inicial macro para as primeiras fases de desenvolvimento. Esse planejamento inicial é feito baseado no escopo do projeto definido, mas deve ser refinado após a Fase 2 em que são fechados os requisitos do projeto. Dessa forma, a Fase 1 passou a se chamar Planejamento Inicial do Projeto. As demais fases permaneceram com mesmo nome.

- Concentrar toda a definição de requisitos (englobando sistemas e software) na Fase 2 de forma a possibilitar um entendimento maior dos requisitos para melhor ser realizado o planejamento do projeto (Fase 3), e;

- Incluir em todas as fases atividades de encerramento da fase, para permitir a criação de baselines, coleta de métricas e registro da situação do projeto.

Até o momento da avaliação, eram pelo menos cinco projetos que faziam uso da segunda versão do processo.

\section{A Avaliação Nível 2}

O processo de avaliação SCAMPI se inicia com uma avaliação prévia denominada Readiness Assessment. A avaliação prévia do CMMI SE/SW do TSE foi marcada para novembro de 2005. Neste momento a CSE/TSE já possuía 11 projetos que utilizavam os processos definidos. Destes 11 projetos, quatro foram selecionados, pelo o líder da avaliação, para o Readiness Assessment: três projetos utilizando a versão 1 do processo e um projeto utilizando a versão 2. Vale ressaltar que a escolha dos quatro projetos avaliados levou em consideração fatores, tais como:

- Ter pelo menos um projeto desenvolvido por cada seção da CSE (SAE, SPE e SVI): para atender a representatividade organizacional desejada;

- Ter projetos com desenvolvimento de software relevante para a organização: no caso do TSE, todos os projetos desenvolveram softwares eleitorais e de apoio à logística das eleições, e;

- O projeto já estar concluído ou em fase de codificação.

\subsection{O Readiness Assesment}

O Readiness ocorreu de 14 a 18 de novembro de 2005. As evidências analisadas neste momento eram os documentos digitais produzidos ao longo de cada projeto que seguiu o processo organizados em uma planilha de evidências disponibilizada pelo líder da avaliação.

Dois representantes da equipe consultora e um representante do TSE (um dos membros do GQPP) participaram do Readiness registrando, principalmente, os pontos de melhoria detectados pela equipe avaliadora. Alguns pontos de melhoria foram: sugestões de formatação de documentos, inclusão de mais informações para auxiliar a padronização, documentação de dados que ainda estavam tácitos, dentre outros. 
Ao final do Readiness todos os pontos de melhorias haviam sido detectados, registrados e foram classificados pela equipe consultora quanto ao seu grau de importância de implementação para atender a prática do CMMI. Com essa informação o líder da avaliação apresentou o resultado ao patrocinador mostrando a viabilidade de realizar os pequenos ajustes para a avaliação oficial e solicitou que fossem incluídas evidências de dois novos projetos que usassem a versão 2 do processo, para mostrar as melhorias que já estavam sendo implementadas.

\subsection{A Avaliação SCAMPI}

A avaliação ocorreu de 12 a 16 de dezembro de 2005. Os objetivos da avaliação SCAMPI definidos pela CSE/TSE foram: (i) comparar a organização com as melhores práticas do mundo; e, (ii) monitorar e controlar o programa de Melhoria do Processo de Software.

Pelo método SCAMPI, apenas os documentos digitais não são suficientes para garantir a evidência de realização de uma prática de um objetivo requerido pelas áreas CMMI. São necessárias também, afirmações, isto é, confirmações dos responsáveis por produzir ou utilizar estes documentos. Assim, na semana da avaliação oficial foram realizadas entrevistas com os líderes de cada projeto avaliado, com os gerentes de cada seção, com os representantes de cada um dos Grupos de Processo (Grupo de Métricas, Grupo de Garantia da Qualidade e Grupo de Gerência de Configuração), com os desenvolvedores e testadores dos projetos. Foram, ainda, realizadas apresentações do processo utilizado pela organização, das ferramentas utilizadas pelos líderes e pelo representante do Grupo de Gerência de Configuração para apoiar a realização das atividades que estavam no processo.

As entrevistas tinham o mesmo objetivo: confirmar que o processo era aplicado na prática por todos os envolvidos; que os documentos eram produzidos no momento correto; que toda a equipe do projeto possuía conhecimento e seguia as atividades pertinentes ao seu papel no projeto. Apenas para ilustrar, um dos projetos avaliados sofreu mudanças constantes de requisitos, já que os requisitos do produto estavam sendo definidos juntamente com alguns representantes de TRE's de várias partes do Brasil. Em particular, este projeto foi uma importante fonte de evidências objetivas para a área de Gerência de Requisitos e a presença de um processo definido auxiliou as várias mudanças de escopo tomando as devidas ações apropriadas e diminuindo o retrabalho. Isto foi relatado por seus participantes.

Ao final da semana, houve o anúncio do resultado do SCAMPI pela equipe avaliadora em que foram destacados os pontos fortes da implantação do programa de melhoria de processo:

- Usar um processo padrão em todas as unidades de desenvolvimento, considerando que isso seria exigido apenas para o nível 3;

- Possuir uma forte definição e gerenciamento de requisitos com a inclusão de grupos de estudo para a definição dos sistemas eleitorais;

- Ter a formalização de testes de software logo após a definição dos requisitos, contribuindo para melhoria da definição de casos de uso, considerando que isso seria exigido apenas para o nível 3. 
- Possuir o comprometimento de todos os envolvidos com a melhoria de processo e a execução do trabalho.

\section{Conclusão e Próximos Passos}

Este artigo apresentou a experiência de implantação de um programa de melhoria de processo de software no Tribunal Superior Eleitoral, englobando desde a definição da estratégia à avaliação final realizada. Todo o trabalho realizado durou pouco mais de seis meses. Segundo os gerentes, líderes e analistas dos projetos envolvidos, dentre as razões do sucesso dessa iniciativa e benefícios alcançados pela implantação de processos na Coordenadoria de Sistemas Eleitorais estão:

- A importância de trabalhar em equipe, respeitando regras e formatos préestabelecidos;

- O entendimento claro da seqüência do desenvolvimento e controle do projeto, sabendo diferenciar a importância necessária de cada atividade;

- A satisfação de transformar a teoria em prática, e ver o resultado, e;

- Aprender realmente a gerenciar um projeto, dando seqüência e alterando todos os documentos que são modificados perante uma decisão.

Esse é apenas o começo do caminho na melhoria de processo de software. O próximo passo para manter a melhoria constante no TSE será a evolução para o nível 3 do CMMI, porém, antes da próxima avaliação SCAMPI, deseja-se alcançar o MPS.BR (Melhoria de Processo do Software Brasileiro) nível E [SOFTEX, 2005].

\section{Agradecimentos}

Aos chefes de Seção (Fábio Trindade, José de Melo Cruz, Marta Medeiros), aos membros do Grupo de Processo, aos líderes e toda a equipe dos projetos, a todos os funcionários da CSE/TSE, e, à Equipe TABA que contribuíram para o sucesso desse projeto.

\section{Referências}

Chrissis, M. B., Konrad, M, Shrum, S. (2003) "CMMI: Guidelines for Process Integration and Product Improvement", Addison-Wesley.

"ISO/IEC 12207:1998 - Information technology - software process life cycle" (1998).

Rocha, A.R., Montoni, M., Santos, G., Oliveira, K., Natali, A.C., Mian, P., Conte, T., Mafra, S., Barreto, A., Albuquerque, A. Figueiredo, S., Soares, A., Bianchi, F., Cabral, R., Dias, A. (2005a), "Fatores de Sucesso e Dificuldades na Implementação de Processos de Software Utilizando o MR-MPS e o CMMI", I Encontro de Implementadores de MPS.BR, Brasília, Brasil.

Rocha, A.R., Montoni, M., Santos, G., Mafra, S., Figueiredo, S., Albuquerque, A., Mian, P., (2005b), "Estação TABA: Uma Infra-estrutura para Implantação do Modelo de Referência para Melhoria de Processo de Software", V Simpósio Brasileiro de Qualidade de Software (SBQS'05), Porto Alegre, Brasil.

SOFTEX, (2005), "MPS.BR - Melhoria de Processo de Software: Guia Geral”, Abril. 